Sessions d'étude - Société canadienne d'histoire de l'Église catholique

\title{
Une oeuvre de la France catholique au Manitoba (1895-1913)
}

Les Chanoinesses Régulières des Cinq Plaies du Sauveur, dites

Soeurs du Sauveur

Antoinette Bergeron

Volume 37, 1970

La vie religieuse au Manitoba

URI : https://id.erudit.org/iderudit/1007280ar

DOI : https://doi.org/10.7202/1007280ar

Aller au sommaire du numéro

Éditeur(s)

Les Éditions Historia Ecclesiæ Catholicæ Canadensis Inc.

ISSN

0318-6172 (imprimé)

1927-7067 (numérique)

Découvrir la revue

Citer cet article

Bergeron, A. (1970). Une oeuvre de la France catholique au Manitoba (1895-1913) : les Chanoinesses Régulières des Cinq Plaies du Sauveur, dites Soeurs du Sauveur. Sessions d'étude - Société canadienne d'histoire de l'Église catholique, 37, 145-164. https://doi.org/10.7202/1007280ar

Tous droits réservés ( $@$ Les Éditions Historia Ecclesiæ Catholicæ Canadensis Inc., 1972
Ce document est protégé par la loi sur le droit d'auteur. L'utilisation des services d'Érudit (y compris la reproduction) est assujettie à sa politique d'utilisation que vous pouvez consulter en ligne.

https://apropos.erudit.org/fr/usagers/politique-dutilisation/ 


\title{
Une cuvre de la France catholique au Manitoba (1895-1913)
}

\author{
Les Chanoinesses Régulières des Cinq Plaies \\ du Sauveur dites Sœurs du Sauveur
}

Il y aura 75 ans le 13 juin, dans six jours donc, qu'arrivaient par train de Montréal, par hatean de Liverpool, trois religieuses françaises à Saint-Boniface. C'étaient Mère Honorine Chanut, Sœur MarieJoseph Bontron et Sœur Marie-Octavie Montet, parties de la Maison Mère de Lyon le 27 mai 1895, à destination de Notre-Dame-de-Lourdes, paroisse manitobaine, fondée en 1891 par le R. P. Dom Paul Benoît, c.r.i.c., et les colons amenés par lui de France et de Suisse. Quelques Canadiens y étaient déjà établis.

Dom Benoît, qui était venu recevoir les trois Chanoinesses à la gare, se hâta de les présenter à $\mathbf{M}^{\mathrm{gr}}$ Adélard Langevin, o.m.i., archevêque de Saint-Boniface, qui les reçut avec joie comme de saintes prémices que Dieu lui envoyait au début de son épiscopat. Il avait été sacré évêque le 19 mars précédent, donc trois mois plus tôt.

C'était la troisième congrégation de religieuses à s'établir dans le diocèse de Saint-Boniface, les Sœurs Grises de la Charité de Montréal étant arrivées en 1844, les Sœurs des Saints Noms de Jésus et de Marie en 1874. C'était la première communauté venant de France. Les trois sœurs demeurèrent cinq jours comme hôtes à la maison vicariale des Sœurs Grises, puis arrivèrent à la gare du Pacifique-Canadien de Rathwell et de là en voiture à Notre-Dame-de-Lourdes, le 18 juin, vers 4. heures de l'après-midi. Bon nombre de paroissiens s'étaient réunis autour de leur pasteur sur la place de l'Église et ils les reçurent dans une grande joie. Le 27 juin, $M^{\text {gr }}$ Langevin vint à Notre-Dame-de-Lourdes pour la confirmation et la première visite pastorale en cette paroisse. Il bénit alors la pauvre maison des sœurs où elles pénétrèrent le lendemain et commencèrent à y vivre cette vie intensément canonique qui les caractérisait comme Chanoinesses Régulières: office divin en commun au complet, chanté en partie les jours de fêtes; lever à minuit pour Matines et Laudes; abstinence de chair tous les jours de l'année; jeûnes du vendredi, et tout le temps de l'Avent et du Carême et les mercredis du 14 septembre à l'Avent et de la Septuagésime au Carême; les douze 
heures de silence sacré de Complies à Primes du lendemain; de 10 à 11 heures du matin; de 2 à 4 heures de l'après-midi.

Dès le premier juillet suivant Mère Honorine et Sœur Marie-Octavie commencèrent à enseigner, la première aux petites filles et la deuxième aux petits garçons, au rez-de-chaussée de leur maison en bois rond de $20^{\prime}$ x 30', Sour Marie-Joseph s'occupant de l'entretien, soit au premier étage où étaient l'oratoire, l'ouvroir et le dortoir, ou dans l'appentis de $12^{\prime} \times 16^{\prime}$ enduit d'argile à peine séché, qui servait de cuisine et de réfectoire.

Pour leurs exercices de piété, la Sainte Messe et l'office du jour les sœurs se rendaient à l'Église où les pères récitaient au chœur l'office en commun à des heures régulières. Elles amenaient leurs quelque trente élèves avec elles pour Vêpres chantées de l'après-midi. Une très grande ferveur régnait dans ce coin de la Montagne Pembina ou s'était formée une collégiale de Chanoines Réguliers sous la direction, on ne peut plus stricte et pieuse à la fois, de Dom Paul Benoît, bras droit du fondateur Dom Adrien Gréa.

Une collégiale de Chanoines Réguliers en pleine forêt - des religieuses de la stricte observance, tout habillées de blanc, portant béguines et rochets, s'adonnant aux jeûnes et au lever de nuit dans une maison si froide que la respiration de chacune se givrait la nuit et les matins d'hiver et dans laquelle l'eau pénétrait les jours de pluie! Quel zèle, quelle ardeur, quelle ferveur! Comment l'expliquer?

Lorsque à la fin du XVIII siècle la révolution française égorgeait les évêques et les prêtres fidèles ou qu'elle les bannissait sur des terres étrangères, elle ne s'attendait guère à réveiller le catholicisme et à l'étendre ailleurs. Tel est pourtant le résultat final de ces persécutions ${ }^{1}$, nous dit l'historien Rohrbacher.

«Puis. ajoute-t-il. la chute de Napoléon (1815), la chute des Bourbons (1830), la chute des Orléans (1848), au moment où les uns et les autres paraissaient les mieux établis furent des grâces extérieures qui contribuèrent à faire réfléchir les Français sur les contingences des valeurs temporelles. L'Église de Dieu que les uns et les autres voulaient rendre plus ou moins leur servante demeurait après eux toujours la même, toujours ancienne et toujours nouvelle. régnant comme une bonne mère dans les esprits et les cœurs ${ }^{2}$."

1 Abbé Rohrbacher, Histoire universelle de l'Eglise catholique, tome vingthuitième, p. 206.

2 Ibid., p. 223. 
On ne pouvait songer à s'appuyer sur la déesse Raison, on ne pouvait compter sur les princes - le gallicanisme sonnait faux à la lumière des événements récents — on s'appuierait sur le roc de Pierre. Les renversements politiques de la première partie du $\mathrm{XIX}^{\mathrm{e}}$ siècle en France apportèrent à la deuxième partie du siècle une recrudescence de l'esprit religieux, de l'attachement au pape et l'esprit missionnaire. $\mathbf{M}^{\text {gr }}$ Léon Christiani, dans sa vie de saint Jean-Marie Vianney, parle de l'ambition qu'avait le jeune curé d'Ars, " de faire reprendre racine à ses paroissiens dans ce bon terreau chrétien de la "Vieille France», et d'ajouter l'auteur: "Nous saisissons ici sur le vif ce bel essor de restoration religieuse qui se poursuit alors par d'ardentes missions, dans toute la France et qui consistait à revenir, par delà les ravages affreux de la Révolution, aux saines et robustes traditions nationales, ou comme disait Joseph de Maistre, précisément à cette époque même (1823), à la «vocation de la France ${ }^{3}$."

Avant d'écrire "que toute souveraineté dont le doigt efficace du grand Pontife de Rome n'a pas touché le front demeurera toujours inférieure aux autres ${ }^{4}$ ", le Comte de Maistre, dans la préface de son livre $D u$ Pape, écrivait: "Il y a des nations privilégiées qui ont une mission dans ce monde. J'ai tâché d'expliquer celle de la France qui me paraît aussi visible que le soleil. Il y a dans le gouvernement naturel et dans les idées nationales du peuple français je ne sais quel élément théocratique et religieux qui se trouve toujours; s'il en manque il n'est pas seulement affaibli, il est mutilé ${ }^{5}$."

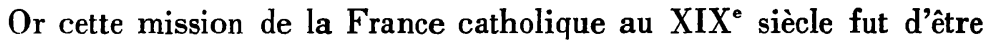
attentive à la voix des papes Grégoire XVI, Pie IX, Léon XIII, l'appelant à fonder des congrégations religieuses pour l'éducation chrétienne de la jeunesse, l'exercice des œuvres de miséricorde, et la contemplation; à établir des associations pieuses et confréries pour secourir le prochain par les saintes aumônes et la prière; à répandre la dévotion à la sainte humanité du Rédempteur, surtout dans ses mystères douloureux; et à répandre la foi catholique dans l'univers par les œuvres missionnaires.

Lyon, cité de saint Irénée et de sainte Blandine, devint particulièrement un centre de ferveur catholique et missionnaire. C'est une humble Lyonnaise, Pauline-Marie Jaricot, qui fonda en 1822 l'œuvre de la Propagation de la Foi qui prit une extension remarquable à travers le monde. Ars est à proximité de Lyon - une trentaine de milles séparant la grande ville du petit bourg. Or c'est par milliers ${ }^{6}$ que les pèlerins

$3 \quad \mathrm{M}^{\mathrm{gr}}$ Léon Christiani, Saint Jean-Marie Vianney, Curé d'Ars, Paris, Apostolat de la Presse, 1954, p. 61.

4 J. de Maistre, Du Pape, cité par Rohrbacher, op. cit., p. 235.

5 Ibid.

6 En 1852 , on estimait à 20,000 le nombre de pèlerins; en 1859 , à 80,000 (Christiani, op. cit., p. 95). 
se rendaient à Ars pour entendre le saint curé, se confesser à lui, se convertir. L'oasis spirituel s'étendit. Un mouvement d'une extraordinaire fécondité surnaturelle se répandit par toute la France et se propagea au dehors. L'humble congrégation que je représente provient de cette poussée spirituelle du milieu du siècle dernier en France, et notamment de Lyon. J'en retracerai brièvement l'histoire.

En 1833, sur les conseils du Curé d'Ars, une pieuse association s'était formée pour honorer d'un culte particulier les Cinq Plaies de Notre Sauveur. Cinq pieuses Lyonnaises, ayant à leur tête Madame Voillot, formèrent le noyau premier de l'Association. Pauline-Marie Jaricot se joignit bientôt à ce groupe.

Le Curé d'Ars, accablé par la foule des pèlerins, ne crut pas pouvoir conserver la direction de l'œuvre naissante. En 1843, il en confia le soin à un prêtre dont il connaissait la pieuse renommée, monsieur l'abbé Colomb de Gast, du diocèse de Lyon, et, sous sa direction éclairée, l'Association s'organisa et se répandit rapidement. Le but des associés était de rendre aux Cinq Plaies de Notre Seigneur le culte qu'elles méritent et d'honorer en même temps les trente-trois années que le Christ a passées sur la terre et, par là, rendre hommage au mystère de la Rédemption commencé à la crèche et consommé sur la croix.

Selon la prédiction du Curé d'Ars les adhérents accoururent en foule; au bout de trois ans les associés étaient au nombre de 10,000. Soumise dès ses premiers pas à l'autorité diocésaine et encouragée par elle, cette pieuse union fut approuvée en 1843 par Son Éminence le cardinal de Bonald, archevêque de Lyon. Le 28 mars 1846, le souverain pontife Grégoire XVI la favorisait de trois Brefs d'Indulgences.

Une souscription volontaire parmi les associés et les dons de quelques généreux bienfaiteurs permirent au directeur d'acquérir en 1856 une ancienne chapelle située à la Croix-Rousse, 65, rue de l'Enfance, Lyon. La chapelle votive fut dédiée aux Cinq Plaies du Sauveur et l'Association y fut canoniquement érigée.

Dès 1857, quelques associées se réunirent à l'ombre de la chapelle des Cinq Plaies pour y mener en commun une vie plus retirée et plus chrétienne. Elles avaient la garde et l'entretien du sanctuaire. tenaient le Bureau de l'Association et recevaient les associés étrangers. Elles recueillaient aussi et élevaient de jeunes orphelines. Ce groupe d'associées était connu sous le nom de "Dévouées". Elles formèrent le noyau premier de la Congrégation des religieuses des Cinq Plaies. Tandis que l'Association s'étendait au loin, ce centre spirituel et temporel, sous le zèle éclairé de monsieur l'abbé Colomb de Gast, se fortifiait et se développait de manière à réaliser au cœur de l'archiconfrérie aussi parfaitement que possible le but et l'esprit de l'CEuvre. 
Le fondateur avait donné aux "Dévouées ", dès le début, l'Écriture sainte comme base de leur vie spirituelle. La vie liturgique était en grand honneur et les "Dévouées » la vivaient avec l'Église. Tous les jours elles se réunissaient au chœur pour la récitation de l'Office canonial. L'étude de la sainte Écriture et des chants sacrés était l'objet de leur soin particulier. Elles faisaient de jour et de nuit, dans la chapelle des Cinq Plaies, l'adoration perpétuelle du Très Saint-Sacrement à l'intention des associés et des bienfaiteurs.

Avant de mourir, le fondateur avait prié le cardinal Caverot, archevêque de Lyon, de vouloir bien lui donner pour successeur son fils spirituel très cher et son aide précieux dans cette œuvre, monsieur l'abbé Auguste Delaroche. Le cardinal accéda à son désir en nommant monsieur l'abbé Delaroche aumônier de la Communauté des Cinq Plaies en 1882 .

Bientôt après la mort du fondateur, le nouvel aumônier conçut le dessein de transformer les "Dévouées " dont il était chargé en congrégation religieuse. Pour la rédaction de leurs constitutions, il s'inspira de la Règle de saint Augustin et des Constitutions des anciennes Chanoinesses Régulières. Le cardinal Caverot l'encouragea fortement dans cette voie, car la communauté constituée par l'abbé Colomb de Gast était déjà, par son esprit et l'ensemble de ses observances, toute préparée à cette vie canonique. Les Constitutions furent approuvées par Son Éminence en 1885, et l'année suivante trente-deux "Dévouées " firent leurs vœux perpétuels. A partir de ce jour, les sœurs portèrent officiellement le nom de CHANOINESSES RÉGULIÈRES DES CINQ PLAIES DU SAUVEUR ?

En 1889, l'abbé Auguste Delaroche entra chez les Chanoines Réguliers de l'Immaculée Conception fondé par Dom Gréa pour restaurer la vie canonique en paroisse. C'est dans la personne du père Augustin Delaroche que la vie des deux communautés fut désormais liée en France comme elle le sera bientôt au Canada.

Cette même année 1889, elles ouvrirent une maison à Saint-Antoine en Dauphiné, diocèse de Grenoble, où les Chanoines avaient leur noviciat. Sept religieuses s'occupaient de la lingerie et de la cuisine des pères.

En 1895. trois sœurs étaient désignées pour la fondation de NotreDame-de-Lourdes au Manitoba. Sœur Honorine de la Croix, "qui depuis quatre ans sollicitait de Notre-Dame la grâce d'aller se dévouer auprès de sa nouvelle église du Canada ", fut désignée pour présider la fondation. Elles furent reçues de grand cœur par Dom Paul Benoît et c'est avec une ardeur correspondante qu'elles entrèrent dans son œuvre.

7 Notes préparées à Lyon pour le centenaire de l'Association des Cinq Plaies en 1943. Archives de la Maison Mère des Sœurs du Sauveur (MMSS). 
De par la volonté des autorités de Lyon, les sœurs du Manitoba devaient entrer dans l'œuvre, et dans la mentalité de l'œuvre de Dom Benoît. Nous présentons deux documents historiques qui le prouvent. Le premier est l'acte d'obédience des trois fondatrices manitobaines. Le second et le troisième, deux lettres de Dom Benoît écrites, l'une à $\mathrm{M}^{\mathrm{gr}}$ Couillé, archevêque de Lyon, et l'autre à une association de Dames bienfaitrices de l'œuvre manitobaine à Lyon. Voici le premier document:

En vertu de la sainte obéissance et sous l'autorité de l'Illustrissime et le Révérendissime Seigneur et Père en Jésus-Christ, Monseigneur Pierre Hector Couillé, Archevêque de Lyon.

Mandons à notre très chère Sœur Marie Honorine de la Ste-Croix, dans le siècle, Honorine Chanut, religieuse chorale, professe confirmée de ce cher monastère, de se rendre en la paroisse de Notre Dame de Lourdes, au Diocèse de Saint-Boniface, Manitoba, Canada, pour y établir une maison de notre ordre, selon la demande qui en a été présentée à notre Révérendissime Archevêque et à nous, par le Très Révérend Père Dom Paul Benoît, Chanoine régulier de l'Immaculée Conception et curé de la dite paroisse au nom des habitants de la paroisse et avec l'agrément de sa Grandeur Monseigneur Adélard Langevin, Archevêque de SaintBoniface, demande accueillie favorablement par sa Grandeur Monseigneur l'Archevêque de Lyon, notre supérieur immédiat.

... Dès leur arrivée à Saint-Boniface nos sœurs devront se présenter devant Sa Grandeur $M^{\text {gr }}$ l'Archevêque pour remettre lis lettres de recommandation de notre Révérendissime Seigneur et Père, $\mathbf{M}^{\mathrm{gr}}$ Couillé, lui montrer la présente lettre d'obédience et recevoir ses ordres et sa bénédiction.

A Notre Dame de Lourdes elles habiteront la petite maison qui leur a été préparée par les soins du Révérend Père Dom Paul Benoît et dans laquelle elles vivront selon nos règles et constitutions, sauf quelques exceptions que devront momentanément subir les articles de la vie claustrale et de l'Office Divin. Nos Sours s'occuperont principalement de l'Euvre des Ecoles paroissiales. Nous désirons vivement que Monseigneur l'Archevêque de Saint-Boniface accorde pour Directeur spirituel et pour confesseur ordinaire le Révérend Curé de Notre Dame de Lourdes; sa Grandeur voudra bien en outre leur assigner un confesseur extraordinaire à son choix. La maison de Notre Dame de Lourdes ne sera jusqu'à nouvel ordre, qu'une simple obédience, relevant en tout de ce premier Monastère.

Cependant à cause de la grande distance qui sépare cette obédience de la maison-mère, nous instituons notre chère Sœur Honorine de la Sainte-Croix Supérieure de cette maison et notre vicaire pour le Canada. En lui conférant les pouvoirs extraordinaires suivants:

Elle pourra $1^{\text {èrement }}$ dispenser les Sœurs de certains points de règle, notamment de l'abstinence et du jeûne d'ordre individuellement, pour un temps qui ne dépassera pas trois mois, et pendant lequel elle nous en référera.

$2^{\text {e }}$ Recevoir des postulantes et après une première probation de six mois les faire canoniquement examiner par le Révérendissime Ordinaire et leur faire donner le Saint habit de la religion.

$3^{\text {e }}$ Faire faire le Noviciat et admettre à la Profession les Sours Converses. Quand aux novices Chorales, elle pourra leur faire faire le postulat et commencer le Noviciat, mais elle devra les envoyer passer au moins un an de leur noviciat et faire leur profession en notre premier 
monastère et il en sera ainsi jusqu'à ce que les conditions requises par nos postulantes étant remplies on puisse ériger la maison de Notre Dame de Lourdes en monastère régulier.

Notre chère Sœur nous rendra compte chaque mois de tous les faits notables qui auront pu se produire dans son obédience et des dispenses qu'elle aura prises, pour elle-même ou accordées aux Sœurs. Tous les six mois elle nous soumettra l'état de son temporel et ne devra faire aucune dépense, prêts ou emprunts de plus de deux cent francs, sans nous avoir consulté.

Et nous prions le Père des miséricordes de bénir cette nouvelle fondation entreprise uniquement pour sa gloire et de la faire prospérer si tel est son bon plaisir. Nous recommandons aussi nos très chères Sœurs à la bienveillance des autorités ecclésiastiques, et les prions de leur prêter aide et assistance partout où elles en auront besoin.

A Lyon, en notre premier monastère des Cinq Plaies de notre Sauveur le 24 mai 1895 fête de Notre Dame Auxiliatrice.

Sr. Marie Iouise de l'Im. Concention Prieure

par ordre de Révérende Mère Sr. Marie Thérèse de Jésus, secrétaire ${ }^{8}$

De cette pièce il ressort que c'est bien à la demande du père Dom Benoît que les Chanoinesses sont venues au Manitoba. Que c'est bien à lui surtout qu'elles furent confiées pour la direction de leur vie spirituelle. Que c'est bien pour un service d'église en paroisse que venaient les sœurs et bien par-dessus tout pour l'éducation de la jeunesse en collaboration avec les autorités paroissiales.

Il ressort aussi que la règle devait être strictement observée comme en France et que le premier monastère entendait bien garder un contrôle très suivi par: (1) la formation des novices chorales, (2) la surveillance des dépenses, prêts et emprunts ne dépassant pas quarante dollars, et un rapport financier tous les six mois, (3) le contrôle des dispenses à la règle du jeûne et de l'office divin tous les trois mois et des faits importants tous les mois. Peu d'autonomie est donnée à la maison du Manitoba qui devait graviter entre ces deux pôles: le monastère de Lyon et les autorités ecclésiastiques locales, la paroisse et le diocèse. Conformément à la mentalité du XIX" siècle, la religieuse avait peu à réfléchir pour la conduite de sa vie, tout étant décidé en haut lieu. La supérieure elle-même avait très peu de jeu dans cette société fortement hiérarchisée. Le souci principal d'une Mère Honorine ne serait pas de prendre telle ou telle décision mais de juger quelle autorité avait la compétence pour la prendre. Tant que les buts, et les moyens pour parvenir à ces buts, furent identiques au monastère de Lyon et dans la paroisse de NotreDame-de-Lourdes, tout alla bien.

Le père Dom Benoît était on ne peut plus heureux d'avoir les sœurs. Il écrit à $\mathrm{M}^{\mathrm{kr}}$ Couillé peu après leur arrivée:

8 Ibid., MMSS: Cahier $\mathrm{n}^{\mathrm{0}}$ 1: Correspondance Monastère des Cinq Plaies à Lyon avec nos premières Sours du Canada, 1895-1913. Boîte $n^{\circ} 43$. 
...Permettez-moi d'exprimer à votre grandeur ma profonde reconnaissance et celle de tous les paroissiens pour l'immense faveur qu'elle nous a faite en nous donnant les Chanoinesses Régulières des Cinq Plaies! Les trois religieuses sont arrivées à St-Boniface le 13 juin et à Notre Dame de Lourdes le 18; elles ont commencé l'école le $1^{\text {er }}$ juillet. Elles prendront un mois de vacances du 15 août au 15 septembre puis se remettront à leur tâche pour une année.

Sa grandeur Monseigneur Langevin, notre éminent archevêque, durant la visite de trois jours qu'il a daigné nous faire, a visité plusieurs fois les Sœurs et a béni solennellement leur monastère avec l'encens, en présence de tout le peuple. Non content de leur promettre d'aller voir Votre Grandeur dans son prochain voyage d'Europe pour la remercier de vive-voix il a du haut de la chaire, en présence de la paroisse et en son nom adressé de ferventes actions de grâces au Primat des Gaules pour le riche don qu'il venait de faire à son archidiocèse. Au Canada une paroisse paraît définitivement fondée quand elle possède l'Eglise et le couvent. Aussi toute paroisse ancienne a des Sours. Par le bienfait de Votre Grandeur, Notre Dame de Lourdes vient de prendre rang malgré sa grande jeunesse parmi les paroisses pleinement établies... C'est la cité de Lyon qui nous a fait ce don, la ville de bonnes auvres, celle qui est en tête de toutes les autres pour l'œuvre de la propagation de la foi, relle qui envoie le plus de missionnaires dans le monde entier. Que Notre Dame de Fourvière soit bénie de sa générosité envers Notre Dame de Lourdes!

En 1639 la France envoyait au Canada ses premières religieuses; c'était déjà des chanoinesses régulières parties de Dieppe et qui tiennent encore l'Hôtel-Dieu de Québec fondé par elles. Depuis, la France a envoyé au Canada beaucoup d'autres religieuses; elle vient de lui donner encore des chanoinesses régulières. Nous espérons que les dernières venues auront les mêmes bénédictions que les premières. Deux curés voisins, les curés de Saint-Léon et de Saint-Alphonse, ont l'intention de s'adresser à Votre Grandeur pour obtenir des mêmes Sœurs. Peut-être notre Archevêque sera-t-il chargé de porter leurs vœux à Votre Grandeur et de les appuyer de sa haute autorité. En tous cas, il est probable que les Sœurs envoyées par Votre Grandeur se multiplieront.

Au Canada en effet, toutes les communautés de femmes, sans exception, se développent merveilleusement ${ }^{9}$...

Les sœurs n'ont pas pris de temps pour se mettre à leur œuvre principale de l'éducation de la jeunesse, treize jours seulement (18 juin au $1^{\text {er }}$ juillet). Le père Dom Benoît est très content de ses religieuses et il en demande déjà d'autres pour Saint-Léon et Saint-Alphonse; en tous cas. il prépare $\mathrm{M}^{\mathrm{gr}}$ Couillé à la demande que lui fera $\mathrm{M}^{\mathrm{gr}}$ Langevin pour des sœurs du même ordre pour les écoles paroissiales. Dom Benoît croit que le recrutement sera facile et que la communauté se développera rapidement au Canada. Il se félicite que ce sont des Chanoinesses Régulières qu'il a obtenues pour ses écoles. Il ne semble aucunement venir à l'idée de Dom Benoît que les Chanoinesses de la stricte observance puissent avoir de la difficulté à combiner harmonieusement l'éducation de la jeunesse au Canada et la vie monastique à laquelle elles se sont vouées: lever de nuit. office canonial entier. abstinence totale et jeûnes.

Examinons une partie d'une autre pièce - une lettre de Dom Benoît à des dames bienfaitrices de Lyon. En préambule. il remercie

9 MMSS. Minuscrit, Cahier $n^{\circ} 6$, p. 3-5. 
ces dames pour les objets envoyés qu'il nomme (ce sont surtout des articles du culte). Puis il ajoute:

La ville de Lyon nous a fait une aumône singulièrement précieuse; c'est l'envoi de trois religieuses des Cinq Plaies pour tenir notre école principale, et plus tard se dévouer aux autres œuvres de miséricorde. Au Canada la plupart des femmes ont passé par les couvents - un très grand nombre d'entre elles comme pensionnaires: De là l'esprit profondément chrétien et extraordinairement distingué de la femme canadienne. De là encore le désir commun de toutes les mères d'envoyer quelque temps, au moins une année ou deux, toutes leurs filles dans une école tenue par les religieuses aussi une paroisse n'est pas regardée comme fondée tant qu'elle ne possède pas son couvent...

...Déjà plusieurs vocations religieuses se présentent; selon toutes les apparences il faudra promptement agrandir la maison que nous avons donné aux Sœurs...

\section{Et le père Dom Benoît intime à ces dames bienfaitrices son rêve:}

... Nous desservons toute une vaste région avec au centre l'église paroissiale de Notre Dame de Lourdes où est chanté tout l'office du jour et de la nuit, où le service est fait avec une splendeur digne d'une grande cathédrale; de tous les côtés sont des centres que nous visitons souvent et que nous voudrions multiplier parce que chacun de ces centres sera plus tard, c'est à dire bientôt une paroisse donnant à l'Eglise des religieux et des religieuses et au ciel des élus. Au centre nous voudrions pouvoir non seulement nourrir les jeunes religieux que nous y avons présentement mais en augmenter le nombre. Beaucoup d'enfants nous sont offerts; faute de ressources nous ne pouvons en entretenir qu'un très petit nombre nous y voudrions pouvoir en recevoir une douzaine, leur donnant l'habit religieux et les élever loin du monde à l'ombre des autels. Notre dessein est lorsque nous serons fortifiés d'envoyer des colonies soit de qq points du gd Nord-Ouest Canadien soit aux EtatsUnis, soit au Japon et en Chine. Tous les premiers explorateurs du Canada y ont cherché la route; en y venant nous aussi avons cru y faire une première étape vers ces 400 millions d'infidèles dont les anges s'adressent à toutes les maisons des religieux pour avoir des missionnaires $10 .$.

Il apparaît nettement que le père Dom Benoît nourrissait d'immenses rêves pour la gloire de Dieu et qu'il voulait que les Chanoinesses y participassent pleinement. Il rêvait aussi d'expansion française en Amérique. A cet effet il ajoute:

... Nous continuons en ce pays de grand avenir notre œuvre catholique et patriotique... Nos Sæurs suivent les Pères dans toutes les fondations et y établissent une école avec un petit pensionnat pour les enfants éloignés. De toutes parts se forment peu à peu des paroisses de langue française qui béniront de siècle en siècle, nous l'espérons nos bienfaitrices de Lyon, leurs généreuses fondatrices $11 .$. .

$10 \quad$ Ibid., p. 10-11.

11 Ibid. Les sœurs sont restées fidèles à la transmission de l'esprit catholique et français dans les écoles. En juin 1939, Mère Augustine était décorée de la France, faite officier d'Académie par M. Raoul Duval, Consul de France à Los Angeles, dans une cérémonie touchante. Elle répondit par ces mots: *A l'exemple des premières religieuses qui arrivèrent au Canada au début de la colonie nous continuerons de faire aimer la France, cette terre classique de l'héroïsme chrétien, transmettant à la jeunesse avec les plus pures notions de la foi, la beauté et l'intégrité de la langue française, (allocution de Mère Augustine, 25 juin 1939, MMSS, Chroniques). 
De 1895 à 1910, les sœurs vaquèrent ainsi dans la ferveur et la joie à leur vie de prières et de sacrifices et avec non moins de zèle à leur vie apostolique. Elles reçurent en ces années une quinzaine de postulantes et eurent la permission de leur faire faire le noviciat, et de nouvelles sœurs arrivèrent de France, une huitaine en tout. de façon à ce qu'elles purent faire trois fondations manitobaines:

Saint-Léon en 1897;
Saint-Alphonse en 1899;
Saint-Claude en 1901.

Les sœurs vaquaient avec dévouement à la tâche de l'éducation et étaient reconnues comme aides précieuses du clergé paroissial, ne négligeant rien qui pût aider celui-ci dans l'accomplissement de sa mission. Les sours étaient dirigées de très près par le père Dom Benoît qui les incitait à être bien fidèles à la règle vouée à leur profession: elles étaient encouragées par l'archevêque de Saint-Boniface qui affectionnait grandement la petite communauté et la soutenait par de nombreuses visites et une direction assez suivie par correspondance avec la Supérieure. Il les aidait et protégeait surtout en ce qui regardait les lois scolaires avec lesquelles elles étaient peu familières. Elles recouraient facilement à lui dans toutes leurs difficultés scolaires comme le témoigne cette lettre de Mère Honorine du $\mathrm{l}^{\text {er }}$ octobre, l'an 1900:

... Nous prenons la liberté d'exprimer à Votre Grandeur la gêne où nous met la Déclaration ou le serment que nous avons à faire tous les six mois en rapport avec le rapport du régistre scolaire.

Nous ne demandons pas en général à enseigner le catéchisme plus qu'une demi-heure par jour mais nous demandons instamment à pouvoir mettre l'esprit chrétien, comme un sel divin, dans tout notre enseignement, à pouvoir expliquer à l'occasion des choses que nous enseignons ou en réponse aux interrogations des enfants, ce qui concerne Dieu, JésusChrist, l'Eglise et tous les dogmes catholiques.

.... N'est-il pas anormal, que n'ayant jamais eu un seul enfant protestant depuis cinq ans que nous tenons l'école, il nous faille nous taire sur la Religion catholique, comme si nos enfants étaient de religion diverse?

Nous savons, Monseigneur combien Votre Grandeur est soucieuse de la liberté de nos écoles. C'est pourquoi nous avons la confiance qu'elle emploiera toute son influence à faire changer un état de chose qui attente à l'équité naturelle, aux droits de nos familles et de nos enfants catholiques, comme aux droits de Dieu et du Sauveur.

Et la réponse de $\mathrm{M}^{\mathrm{gr}}$ Langevin ne se fait pas attendre. Elle est de son secrétaire, Arthur Béliveau, et datée du 9 octobre 1900.

... Cette formule est une restriction odieuse et injuste de la liberté de lenseignement religieux, à laquelle nous avons droit et si une interprétation libérale nous permet d'en éluder la rigoureuse application, elle n'en reste pas moins une atteinte à la liberté de conscience et elle peut parfois occasionner des ennuis sérieux. Sa Grandeur désire donc que vous lui adressiez au plus vite une lettre conjointe signée par vos maîtresses de classe et exprimant la gêne que vous cause cette formule 
de déclaration équivalent à un Serment consignée dans le rapport semestriel que vous avez à signer ${ }^{12}$.

De Lyon elles avaient régulièrement des lettres qui leur apportait l'esprit de la Maison des Cinq Plaies dans toute la ferveur de ses débuts et l'affection de leurs sœurs dans la chaleur du "Cor Unum Anima Una », devise de l'Association des Cinq Plaies. La révérende Mère Marie-Louise visitait à tous les six ans ses maisons du Canada pour deux ou trois mois, apportant à toutes ses sœurs l'affection de son grand cour et des nouvelles de toutes leurs familles particulières et surtout de leur famille religieuse, de son développement et rayonnement.

\section{IV}

Jusqu'en 1906 au moins, il y eut complète harmonie entre SaintAntoine - Lourdes - Lyon - Saint-Boniface. Le rêve de Dom Benoît exprimé en 1878 se réalisait. Il avait écrit à ce temps:

Nous nous destinons aux missions étrangères. Mais nous reprendrons l'ancienne méthode d'évangélisation: celle des monastères. Notre fondateur a reçu du Bon Dieu beaucoup de lumière sur ce point. D'après ces vues le monastère est le vrai moyen de former un clergé indigène. en offrant à l'inconstance des pays infidèles les secours de la vie de communauté et des vœux ${ }^{13}$.

$\mathrm{M}^{\mathrm{gr}}$ Langevin, de son côté, était ravi du bien opéré par l'équipe de Dom Benoît. Il écrivait à Dom Gréa le 24 janvier 1896:

Quel bien immense! Voilà un pays qui sera bientôt complètement catholique et français, grâce aux efforts intelligents et aux sacrifices héroïques de votre pieuse congrégation! Le digne Père Dom Benoît est admirable à la tête de sa petite phalange si gaie et si fervente. C'est un homme de Dieu, qui a le génie des vieux moines colonisateurs du moyen âge. Une vertu divine est sortie de la France par cette grande et belle âme, et elle opère des prodiges ici ${ }^{14}$.

La formule de recrutement et de formation que Dom Benoît voulait pour la communauté des pères, il la voulait aussi pour celle des Chanoinesses. Les sœurs avaient établi des pensionnats dans chacune des paroisses où elles formaient à la vie intérieure des jeunes filles d'une façon assez analogue à celle que donnait les pères aux petits frères.

Mais vers 1906, l'œuvre de Dom Gréa était remise en question par un groupe de Chanoines dont le père Augustin Delaroche. maître des novices et prieur claustral d'Andora. Certains événements douloureux pour les sœurs dont il avait été longtemps le Supérieur ${ }^{15}$ le faisaient

12 MMSS, Manuscrit, Cahier $\mathrm{n}^{\circ}$ 6, p. 39.

$13 M^{\mathrm{gr}}$ Vernet, Dom Gréa, Labergerie, p. 120.

14 Il est souvent question des Chanoines Réguliers et de l'admiration de $\mathrm{M}^{\mathrm{gr}}$ Langevin pour Dom Gréa et Dom Benoît dans la Vie de $M^{g r}$ Langevin (18551915) par le R.P. Morice, o.m.i., Saint-Boniface, 1916.

15 De 1882 à 1899. A partir de 1899 par la Constitution • Conditæ », l'évêque où était la Maison Mère d'une communauté diocésaine n'avait pas plus de pouvoirs que les autres évêques qui avaient des sœurs du même ordre dans leurs diocèses, 
réfléchir profondément sur la sagesse des grandes rigueurs à elles imposées par leur règle, car au temps des décrets d'expulsion des religieux en France de 1901 à 1903 la Communauté des Cinq Plaies avait dû prendre le chemin de l'exil. La Maison Générale et le noviciat s'étaient réfugiés à Diano-Marina dans le diocèse d'Albenga en Italie.

L'exil était cruel. L'extrême pauvreté et les durs travaux étaient très lourds pour la petite communauté. La mort moissonnait dans ses rangs de belles gerbes que les recrues locales ne parvenaient pas à remplacer. Une quinzaine de religieuses étaient mortes à la fleur de l'âge.

Le cardinal Vivès. cardinal protecteur des Chanoines à Rome et qui devait bientôt devenir président de la Sacrée Congrégation des Religieux, prit la chose en main et, par la Constitution promulguée le 11 octobre 1908. la Congrégation des Chanoines Réguliers fut transformée au point où le fondateur disait ne plus reconnaître son œuvre ${ }^{16}$. On demandait à celui-ci d'abandonner à son vicaire général. Dom Delaroche. le gouvernement de l'institut.

Plusieurs pères de Suisse, de l'Est et de l'Ouest canadiens, quittèrent à ce moment la congrégation et entrèrent dans le clergé séculier. Les dix religieux du Manitoba. la mort ${ }^{1 i}$ dans l'âme. par ce qu'ils crurent être "fidélité au Fondateur ${ }^{18}$ " et fortement conseillés dans ce sens par l'archevêque de Saint-Boniface ${ }^{19}$. se sécularisèrent en 1912.

Dans l'intervalle, précisément en août 1910, alors que les pères étaient dans l'hésitation et l'angoisse ${ }^{20}$, la révérende Mère Marie-Louise. supérieure générale des Chanoinesses vint de Diano-Marina faire sa

et ne pouvait plus déléguer son pouvoir à un prêtre du diocèse qui serait leur supérieur. Conséquemment, le R.P. A. Delaroche cessa d'être le supérieur des Chanoinesses le 8 décembre 1899 (détails obtenus d'une lettre de Dom Benoît à Mère Augustine, 25 janvier 1912). Les liens de Dom Delaroche avec la communauté des Chanoinesses n'en restèrent pas moins, on peut bien le croire, extrêmement forts.

16 MMSS, Lettre de Dom Gréa à $M^{\mathrm{gr}}$ Langevin, 27 octobre 1908: . ...la nouvelle constitution qui abolit notre æuvre dans ses points essentiels ", cité par $M^{\mathrm{gr}}$ VERNET, op. cit., p. 156.

17 MMSS, Dom Paul Benoît, carnet intime inédit, $\mathrm{n}^{\circ}$ 111, au 9 octobre 1909: - Je dis au P. Delaroche: "Je suis comp. désespéré; on n. a enlevé notre père; on n. a enlevé notre sainte vie. Si vous venez pour achever de n. détruire, et bien, faites-le. Mais ce que v. feriez mieux de faire, ce serait de rebrousser chemin et de tout restaurer.

18 MMSS, Dom Benô̂T, op. cit., 15 août 1909: * Je fais væu de fidélité au fondateur et à son ouvre, vœu de ne jamais l'abandonner et d'employer tous les moyens légitimes pour la rétablir. " Au 22 mars 1910: «... Nous n'accepterons la sécularisation que pour demeurer fidèles à l'œuvre de notre Fondateur. "

19 Ibid., au 19 juillet 1909: $\mathrm{M}^{\mathrm{gr}} \mathrm{m}$ 'a dit plusieurs fois que $\mathrm{n}$. avions mal fait de ne pas suivre son conseil de n. séculariser, que n.n. en repentirions et qu'il ne pouvait plus nous aider. .

20 Le 12 janvier 1910 le cardinal Vivès avait envoyé une lettre à Dom Benoît supprimant l'école de théologie, et obligeant Dom Benoît à se démettre du supériorat et de se rendre à Saint-Léon pour Pâques. (MIISS, carnet intime, $\left.\mathrm{n}^{\circ} 111.\right)$ 
visite à Notre-Dame-de-Lourdes et apporta des adoucissements considérables à la règle suivie jusqu'ici par les sœurs; changements très semblables à ceux proposés aux pères dans leurs nouvelles constitutions: Matines et Laudes le soir au lieu de la nuit; chant de Vêpres le dimanche et les fêtes de $\mathrm{l}^{\text {re }}$ classe seulement; soeurs enseignantes dispensées de l'office choral les jours de classes; jeûnes de l'Église et quelques autres jours seulement; l'abstinence prescrite par l'Église et 2 jours par semaine dans l'Avent et 3 jours en Carême ${ }^{21}$.

Dans une lettre du 3 février précédent Mère Marie-Louise avait demandé aux sœurs du Canada d'indiquer "les difficultés qu'elles rencontraient pour l'observance de la règle, en raison de vos auvres, du climat, et d'un personnel trop restreint ${ }^{22}$ ", et les sœurs du Manitoba avaient répondu que tout était bien, qu'elles ne voulaient pas d'adoucissements à là iégle qu'elles avaient vouée en 1886. Et à partir de ce temps les sœurs récitaient tous les jours après l'élévation - tout fort le psaume Levavi pour obtenir la séparation ${ }^{23}$.

Mère Marie-Louise avait intimé à Mère Honorine de faire mettre à exécution ces changements sans en entretenir $\mathrm{M}^{\mathrm{gr}}$ Langevin et le père Dom Benoît, et " il n'y a pas à s'inquiéter de quelques modifications qu'une haute prudence inspire et que le devoir des Supérieurs (le père Augustin Delaroche, et elle-même et son Conseil) est de mettre les Règles de leur institut d'accord avec les normes ${ }^{24}$ ».

Mère Honorine avait déjà à cette époque une santé chancelante. Le conflit d'allégeance entre ses supérieures de France et ses directeurs et Supérieur ecclésiastique du Canada l'atterra. Elle mourut le 8 septembre suivant. "Cette femme à l'intelligence remarquable, à l'âme virile. au cour merveilleusement tendre », dit d'elle le père Dom Benoît, dans une Vie de Mère Honorine encore inédite; cette âme, "la plus contemplative de mon diocèse ", disait d'elle $\mathbf{M}^{\mathrm{gr}}$ Langevin, emporta dans la tombe le secret des changements apportés à la règle un mois plus tôt, comme aussi le secret de sa douleur partagée avec nul autre, semble-t-il, que Jésus crucifié.

La révérende Mère Augustine, femme remarquable par sa distinction, et sa bénignité, était venue de Lyon en 1896, pour la fondation de l'école et du couvent de Saint-Léon cette même année. C'est elle qui le 8 novembre 1910 fut nommée par Lyon pour remplacer Mère Honorine

21 MMSS, * Les deux règles \#, Boîte $n^{\circ} 16$.

22 MMSS, Lettre de Mère Marie-Louise, 3 février 1910.

23 MMSS, *Petites Mémoires *, 30 novembre 1957, Sœur Marie-Elizabeth Comte, la première postulante canadienne. Il semble bien que ceci se faisait sous l'inspiration de Dom Paul Benoit, directeur spirituel de la communauté.

24 MMSS, lettre de Mère Marie-Louise à Mère Augustine, le 29 juin 1912, lui disant de copier les parties changées des constitutions et d'envoyer cela à $\mathbf{M}^{\mathrm{gr}}$ Langevin qui avait entendu parler de changements et s'en inquiétait. 
à la tête de la communauté manitobaine qui comptait à ce temps trente religieuses. Elle prit alors connarssance des changements opérés dans l'institut sans le consentement des supérieures des couvents du Manitoba, et sans consultation préalable avec l'autorité ecclésiastique du diocèse de Saint-Boniface. Elle communiqua à Dom Benoît ces changements et il lui répondit:

$\mathrm{Si}$ on change notablement votre règle vous ferez bien de vous concerter avec vos Sœurs et de prendre les moyens nécessaires pour le maintien de la règle que vous avez pratiquée jusqu'ici. Et le moyen tout providentiel, simple et efficace est l'intervention de l'autorité de votre Archevêque 25.

C'est alors quelle communiqua son émoi à monseigneur l'archevêque au sujet de ce changement radical de leur vie. Il y eut une forte réaction chez $\mathrm{M}^{\mathrm{pr}}$ Langevin qui ressentait vivement qu'on ne l'eût pas consulté au sujet de ces altérations profondes dans une congrégation de son diocèse qu'il protégeait et aidait sensiblement depuis ses tout débuts. Dom Benoît, le conseiller spirituel premier de la congrégation, pour des raisons différentes ${ }^{26}$, se joignit à $\mathrm{M}^{\mathrm{gr}}$ Langevin pour déplorer le malheur qui frappait les sœurs après les avoir frappés eux-mêmes ${ }^{2 \tau}$. Les souurs se rangèrent toutes de ce côté et demandèrent la protection de l'archevêque de Saint-Boniface. Le 28 février 1912. Mère Augustine écrivit à celui-ci :

...Je me permets de venir réclamer l'appui et le secours que vous moffrez avec tant de bienveillance afin d'obtenir qu'on nous laisse toutes ces observances de notre première Règle ou qu'au moins on prolonge encore quelques années l'expérience. Toutes les Sœurs de notre Maison du Manitoba m'ont exprimé le même vœu... Notre titre de Chanoinesse des Cinq Plaies semble résumer trois obligations inséparables: la louange divine, la pénitence et les œuvres de charité. En conséquence nous tenons à ce qui nous fait essentiellement remplir le but de notre sainte vocation; demandant que l'office de la nuit ne soit pas supprimé, $2^{\circ}$... que la part que nous donnions à la louange Divine ne soit pas diminuée, $3^{\circ}$... que les abstinences et les jeûnes si modérés de notre Règle ne soient pas diminués 28 .

Le 5 février 1913, $\mathbf{M}^{\mathrm{gr}}$ Langevin promulgua l'ordonnance de séparation d'après la Constitution "Conditæ a Christo ${ }^{29}$ » qui fut combattue

25 Lettre de Dom Benoît à Mère Augustine, le 25 janvier 1912 । MIMSS. Boîte $\mathrm{n}^{\circ}$ 16).

26 Le père Dom Benoît était surtout affligé parce que les sœurs perdaient leur vie canonique. $\mathrm{M}^{\mathrm{gr}}$ Langevin parce qu'elles se recruteraient moins au Manitoba si le noviciat devait se faire à Nominingue plutôt qu'à Notre-Dame-de-Lourdes, aussi * parce qu'on n'avait pas été franc avec lui et qu'on avait modifié les constitution en secret "! (Lettre de $\mathbf{M}^{\mathrm{g} 1}$ Langevin à Mère Augustine, 12 avril 1913.)

27 * Les mêmes ouvriers qui ont détruit l'œuvre du $R^{\text {me }}$ Dom Gréa travaillaient sourdement en dehors de moi à satisfaire leur besoin de diminution de la Congrégation des Cinq Plaies... un seul noviciat... et d'un seul lieu de recrutement ( $\left(\mathrm{M}^{\mathrm{gr}}\right.$ Langevin au R.P. Louis Ferrey, le 31 mars 1913; MMSS, Boîte $\left.n^{\circ} 16\right)$.

28 MMSS, lettre de Mère Augustine à $\mathrm{M}^{\mathrm{gr}}$ Langevin, 28 février 1912.

29 MMSS: "J'ai enfin décidé d'user de mon autorité pour sauver les maisons des sceurs des Cinq Plaies de Notre Sauveur et je les ai séparées de la Maison-Mère 
à Rome par tous les moyens. M. Charles Lecoq, prêtre de Saint-Sulpice, fut chargé, le 18 juin 1913, par la Sacrée Congrégation des Religieux pour travailler à la réunion des maisons manitobaines avec la souche de l'institut. Il y travailla quatre mois mais ne put réussir à évoquer des motifs suffisants pour ébranler la volonté de l'archevêque ${ }^{30}$ et des soeurs qui s'étaient rangées définitivement à l'abri de l'autorité ecclésiastique manitobaine. Le 30 octobre 1913 le décret de séparation fut donné à Rome.

Comment expliquer que dans ce conflit d'autorités de la France et du Canada les sœurs se fussent rangées immédiatement avec celles-ci plutôt qu'avec celles-là. La question était-elle vraiment qu'elles ne trouvaient aucunement opportuns ces adoucissements à la règle, alors qu'il était très pénible pour les Canadiennes surtout d'observer la règle dans sa totalité, et que les dispenses étaient nombreuses à ce temps? L'évêque de Saint-Boniface était conscient de ces difficultés — n'avait-il pas cherché lui-même à adoucir la règle ${ }^{31}$ ? Les liens fraternels avec la France et l'Italie extrêmement forts du début s'étaient-ils relâchés à

de Diano-Marina. C'est ma conviction que leur recrutement était menacé, et alors c'était la ruine à courte échéance " (lettre de $\mathbf{M}^{\mathrm{gr}}$ Langevin au $\mathrm{P}$. Antoine Chalumeau, 10 février 1913).

30 MMSS: - Votre Grandeur pardonnera à un Sulpicien s'il aime et préfère ces grandes familles (les familles religieuses de droit diocésain mais qui sont établies dans plusieurs diocèses et dont les évêques doivent s'entendre pour modifier les constitutions, s'il y a lieu de les modifier). La vie de l'Eglise est tellement large, tellement dilatable qu'elle semble aimer ce qui ignore et franchit les limites, ce qui est de la race de Japheth: "Que Dieu donne de l'espace à Japheth; que Japheth habite dans les tentes de Sem., - Les fautes commises (ignorant l'autorité de $M^{\mathbf{g r}}$ Langevin) sont des fautes de personnes, non des vices de l'Ordre. La leçon donnée par les derniers événements ne sera pas perdue. Les religieuses du Manitoba vous sont toutes soumises et unies, Monseigneur, comme le prouve l'acte qu'elles ont unanimement signé. Votre Grandeur n'est-Elle pas sûre d'obtenir tout ce qu'Elle demandera, sans poursuivre l'instance en séparation de corps et de bien. * C'est ce que j'ose humblement solliciter de Votre Grandeur. Qu'Elle daigne retirer sa demande. Qu'Elle obtienne une Province avec toute la part d'autorité qui lui revient. Une telle solution ne donnerait-elle pas satisfaction à tous les deux, au cour surtout de Celui qui s'appelle, l'auteur de la Paix " (lettre de M. Charles Lecoq à $M^{\mathrm{gr}}$ Langevin, le 7 août 1913 , boîte $\mathrm{n}^{\circ} 46$ ).

$31 M^{\text {rr }}$ Langevin écrit à Mère Augustine en 1912: "Avez-vous gardé et observé toute la règle, jusqu'ici ? La Mère Honorine m'a dit que cela n'était pas possible " (lettre du 2 mars 1912); .... vos sœurs ont des classes régulières à faire, avec un enseignement bilingue et comme conséquences de longues préparations pour être à la hauteur des programmes et arriver aux brevets. Vos sœurs destinées à faire leur marque dans l'enseignement ont besoin de beaucoup de temps pour l'étude, si elles veulent être à la hauteur des exigences de notre temps , (lettre de novembre 1912). Au bas de la lettre, au crayon, de la main de Dom Benoît, sont écrits ces mots: - Triste, Triste, Triste - N'abandonnez pas les institutions traditionnelles de votre ordre pour de soi-disant exigences modernes. Cherchez le Royaume de Dieu et sa justice et tout le reste vous sera donné par surcrô̂t = (ibid., MMSS, Boîte $\mathrm{n}^{\circ} 16$ ). 
ce point que les sœurs abandonnassent, avec gaieté de cœur presque, leurs anciennes mères, leurs sœurs bien-aimées ${ }^{32}$ ?

Étaient-elles vraiment bien libres dans leur démarche? Une lettre d'une des trois fondatrices du Manitoba, Sœur Marie-Octavie, nous permet d'en douter. Elle écrit à monseigneur Langevin le 31 mai 1913, donc peu après le décret de séparation:

... Monseigneur, maintenant veuillez permettre à votre humble fille de vous ouvrir son cœur sur un point très délicat. Ayant le cœur très sensible il m'en a bien coûté les premiers jours que nous reçûmes le document et l'ordonnance qui sépare nos maisons de la Maison-Mère de Diano. Il me fallut faire un effort suprême pour ne pas me laisser envahir par la tristesse mais j'ai compris que c'était pour le plus grand bien de notre communauté. C'est bien à vous que nous devons cette résurrection de notre communauté. Le Bon Dieu me fit la grâce de surmonter la tentation d'ennui et d'abandonner toute idée personnelle. Je sais que vous êtes un bon Père, votre zèle pastoral aurait souffert de laisser périr vos auvres 33 .

Les sœurs sont depuis quinze ans au Manitoba profondément liées à l'œuvre des Pères de Notre-Dame-de-Lourdes, associées toutes, on ne peut plus fortement. au rêve de Dom Benoît, cet homme au dynamisme extrêmement puissant, à la vie spirituelle intense et conquérante ${ }^{34}$. Vingt et une des sœurs sont Manitobaines et par conséquent moins attachées à la France. Après l'exil à Diano-Marina, les lettres et chroniques entre les sœurs d'Italie et du Canada tombèrent de trente-trois en 1900 à quatre en 1901. Repliées sur leur angoisse et leurs souffrances nombreuses les sœurs exilées avaient moins de temps et de goût pour des relations avec les sœurs canadiennes. Mais il nous apparaît surtout que les religieuses furent sujettes et victimes d'un conflit d'autorité et qu'elles eurent peu à réfléchir ou ne voulurent point réfléchir et prendre une décision conforme à leur bien personnel et à celui de l'institut. Il y avait peu d'autonomie pour la femme religieuse au début du $\mathrm{XX}^{\bullet}$ siècle, un siècle qui traînait encore l'autoritarisme ultramontain de la fin du

32 Une lettre déchirante fut écrite aux sœurs du Canada par la R. Mère Marie-Louise à l'occasion de la séparation; elle est datée du 16 mars 1913: a... Elle est donc arrivée cette missive qui a été pour mes Sœurs et pour moi un sujet d'immense tristesse. Oh! vous ne pouviez rien écrire qui puisse me causer un plus grand chagrin... Vos lettres toujours bonnes, filiales, affectueuses, n'étaient donc pas l'écho de vos vrais sentiments puisque vous avez voulu rompre si rapidement et sans m'avertir? ... N'auriez-vous donc pas pu attendre quelques années encore que notre Révérend Père Marie Augustin et moi qui avons jeté les fondements des missions canadiennes, que nous ayions l'un et l'autre quitté cette terre afin de nous épargner cette profonde douleur.... (MMSS, Boîte $\left.\mathrm{n}^{\circ} 16\right)$.

Boîte $\mathbf{n}^{\circ} \mathbf{4 3}$.

33 Lettre de Sœur Marie-Octavie à $\mathbf{M}^{\mathrm{gr}}$ Langevin, 31 mai 1913, MMSS.

34 On se soumettait aux conseils du père $D$. Benoît comme à des ordres tant son prestige était grand dans la communauté des sœurs. Il dirigeait de très près Mère Augustine dans ses relations avec l'extérieur comme le prouvent ces lignes: - Je vous prie de les copier [observations sur les changements]; vous en userez ensuite avec Monseigneur comme vous le jugerez à propos; mais changez le plus possible la forme, pour qu'on ne voie pas que j'y suis pour rien. (lettre de Dom Benoît de Saint-Léon à Mère Augustine, 7 février 1912). 
XIX ${ }^{\text {e }}$ Ceci explique peut-être pourquoi les sœurs résistèrent à des changements à la règle, qui paraissent aujourd'hui si raisonnables et qui le paraissaient peut-être pour plusieurs d'entre elles aussi à ce temps, et qu'on dut faire par la suite, mais qui n'étaient pas dans la ligne de spiritualité dans laquelle elles étaient dirigées à ce temps.

Le cardinal Suenens, dans son livre Promotion apostolique, dit de la femme du XIX ${ }^{e}$ siècle, à plus forte raison de la religieuse: "La femme vivait emprisonnée dans une sorte de destin immuable, dans le cadre d'un archétype idéal, fixé une fois pour toutes et imaginé par des hommes. On la voulait docile, fidèle, résignée, laborieuse, mais dans les limites étroites, à l'abri des courants d'air et du vent du large. "

Tel nous apparaît bien l'état d'esprit et de fait de nos religieuses dans la vie de l'Église de France comme dans celle du Manitoba en cette période de l'histoire de la communauté que nous avons décrite (1895 à 1913).

Cette mentalité convenait peu aux œuvres entreprises au Manitoba pour la gloire de Dieu. A une spiritualité purement contemplative aurait dû se superposer progressivement une spiritualité davantage orientée vers l'action. L'équilibre entre la vie de prière et la vie d'apostolat n'était pas réalisé au niveau de la spiritualité même. La perspective contemplative gardait une primauté qui, en certains points, cadrait mal avec les exigences de la vocation active.

Entre 1915 et 1935, on s'employa à conserver les exercices de vie contemplative qu'on avait hérités des Fondateurs et à laquelle avec une apparente générosité on avait sacrifié l'union avec la France en 1913. On essaya en même temps de conserver et de développer les œuvres d'éducation dans les paroisses où elles existaient avec un entier dévouement. Les personnes, surtout les enseignantes, subissaient alors de fortes tensions, tiraillées qu'elles étaient entre deux fidélités - la règle et le devoir d'état.

«Quoi qu'il en soit, les changements qui, en 1910, avaient paru inopportuns, que plusieurs avaient jugés sévèrement et peut-être attribués à un manque de générosité chez les sœurs d'Europe, semblèrent s'imposer après un certain nombre d'années ${ }^{35}$ »

Les santés étaient ébranlées, le nombre d'entrées diminuait ou en tous cas ne progressait pas normalement. Il y avait en 1935 six maisons et cinquante religieuses.

"L'institut des Chanoinesses des Cinq Plaies, monastique dans sa formation première, devait subir une transformation et s'adapter. afin

35 MMSS, Les Chanoinesses des Cinq Plaies du Manitoba, correction, R.P. A. Champagne, 1956. 
de poursuivre une de ses principales œuvres au Manitoba: l'éducation de la jeunesse. Les anciennes qu'étaient nos fondatrices s'étaient épuisées à la tâche et atteignaient tour à tour le terme de leur active et parfois héroïque carrière ${ }^{36} \ldots$.

L'adaptation de la communauté aux conditions nouvelles du monde et de l'Église commença au chapitre général de 1937 et s'est poursuivie constamment depuis. Les religieuses, encouragées par nos évêques, s'appliquèrent à réfléchir par elles-mêmes sur leur vie religieuse et à faire les adaptations nécessaires pour que les personnes puissent trouver à l'intérieur de la communauté l'équilibre entre prière et action et un sain épanouissement.

La tendance contemplative de la spiritualité ne fut pas détruite mais peu à peu libérée des séries de prescriptions canoniques qui tendaient à bloquer l'élan apostolique. L'Action catholique dans nos écoles, les directives de Pie XII aux religieuses, le Concile Vatican II, la Conférence catholique canadienne, l'encouragement de nos évêques manitobains et des liens renoués avec la communauté de France depuis 1950 furent tous des facteurs positifs qui permirent à la communauté d'entrer de plain-pied dans une recherche communautaire fructueuse et de vaquer à son développement personnel à la lumière de son histoire comme aussi des besoins de l'Église du $\mathrm{XX}^{\mathrm{e}}$ siècle. Il nous semble en 1970 avoir accompli la tâche d'adaptation qui s'imposait, i.e. d'avoir donné à la communauté la forme nouvelle qu'exige la percée de l'Évangile du Salut dans le monde qui se construit. En guise d'illustration je vous lirai un chapitre de notre Règle de Vie intitulé: "Vie apostolique des Sœurs du Sauveur "... Il porte sûrement l'empreinte de notre passé, mais il s'insère dans l'époque présente de l'histoire de l'humanité et nous semble s'ouvrir pleinement sur l'avenir.

Sœur Antoinette Bergeron, s.d.s., B.A., B.Éd., M.Éd., Université du Manitoba.

\section{VIE APOSTOLIQUE}

- Nous ne pouvons pas, quant à nous, ne pas publier ce que nous avons vu et entendu : (Ac 4, 20).

29. Dans un unique mouvement d'amour et d'attachement profond à Jésus-Christ, qui, tout en même temps, les conduit à son Père et les envoie à ses frères, les Sœurs du Sauveur se livrent avec égale ardeur à la contemplation et au service fraternel.

36 Ibid. 
AA 3

Jn 3,16

Col 4, 6; 3, 17 PC 20

LG 33

GS 4

Rm 12, 2

Ep 5, 10.15

1 Th 5,21

$\mathrm{Ph} \mathrm{3,20s}$

$1 \mathrm{Jn} \mathrm{3,} 2$

Rm 8, 19.22

Col $1,15-20$

Ep 1, 22.23
30. Elles saisissent

toute occasion, prévue ou imprévue,

et utilisent à bon escient

les moyens à leur disposition

poür éveiller parioui

l'esprit de foi

et pour faire connaître et aimer

Celui

qui a tant aimé le monde.

31. Toujours, elles songent à dire

ce qui convient,

et à faire

ce qui est opportun,

de sorte que leur présence

et leur attitude

puissent témoigner

auprès des hommes

de l'amour que Dieu a pour eux,

et donc les acheminer

vers une rencontre personnelle

avec Lui.

32. Que les Sours s'emploient

à une ouvre bonne et bienfaisante,

qu'elles exercent une compétence professionnelle,

ou qu'elles collaborent aux activités

plus directement liées à la mission

qui incombe aux pasteurs de l'Eglise,

leur service reste toujours un engagement

pour le Christ

parachevant son ouvre de rédemption.

33. Tenant compte des aspirations

des hommes d'aujourd'hui

et devant l'évolution qu'elles voient s'opérer

dans tous les domaines,

elles s'interrogent

et se tiennent de leur temps.

34. Elles admirent les artisans du progrès

et, selon leurs moyens,

elles stimulent et secondent leurs efforts.

Elles s'efforcent, avec discernement,

de garder un sain équilibre

dans leur propre conduite

et dans leur travail

qu'elles veulent efficace

et surtout porteur d'espérance.

Car les Sœurs du Sauveur croient

et annoncent

que l'homme et le monde vont

se renouvelant et se transfigurant

dans le Christ 37. 


\section{BIBLIOGRAPHIE}

\section{I. - Manuscrits et documents dactylographiés}

Benoîr, Dom Paul, Ad suggerendum memoria (25 avril 1901 au 7 novembre 1915), Manuscrit, MMSS, Boîte $n^{\circ} 43$.

- Vie de Révérende Mère Honorine de la Croix (1848-1910), dactylographiée, inédite, Saint-Léon, 1912, MMSS, Boîte $\mathrm{n}^{\circ} 44$.

Chroniques du Couvent Notre-Dame-de-Lourdes (1895-1970), MMSS, Boîte $n^{0} 45$.

Correspondance avec la Maison des Cinq Plaies (1895-1913), Cahier n" 1, MMSS, Boîte $\mathbf{n}^{\circ} 43$.

Correspondance. Evénements 1908-1915, MMSS, Boîte $n^{0} 16$.

- Exposé sur la Fondation de la Congrégation des Chanoinesses des Cinq Plaies, présenté à $\mathrm{M}^{\mathrm{gr}}$ Dupuy, auxiliaire de Lyon, à l'occasion du $100^{\mathrm{e}}$ anniversaire de la Fondation de l'Association des Cinq Plaies» (Archives de Lyon), MMSS, 1956.

- Notes historiques sur l'origine et le développement de l'Institut des Chanoinesses des Cinq Plaies du Sauveur ", Saint-Boniface, 1958, MMSS.

- Petites Mémoires s, compilées par S. L. Préjet, d'entrevues avec nos anciennes sœurs, 30 novembre 1957, MMSS, in folio.

Règle de Vie, Saeurs du Sauveur, Chapitre d'Aggiornamento (1967-1968), publiée à Saint-Boniface, le 9 janvier 1969.

Le sigle MMSS: Archives de la Maison Mère des Sœurs du Sauveur.

$$
\text { II. - Livres }
$$

Cụristiani, $\mathrm{M}^{\mathrm{gr}}$ Léon, Saint Jean-Marie Vianney, Curé d'Ars (1786-1859), Paris, $2^{\circ}$ éd., Apostolat de la Presse, 1954.

RoHRBACHER, l'abbé, Histoire universelle de l'Eglise catholique, Liège, tome vingthuitième (1802-1848), Imprimerie de J. S. Lardinais, éditeur, 1849.

Sunnens, le cardinal Léon-Joseph, Promotion Apostolique de la Religieuse, Bruges, Desclée de Brouwer, 1962.

Vernet, M ${ }^{\text {gr }}$ Félix, Dom Gréa (1828-1917), Paris, Labergerie, 1937. 